\title{
Attack Resistant Digital Image Watermarking using Complex Wavelet Transform
}

\author{
Sukhwinder Kaur Bhatia \\ IKG Punjab Technical University Research \\ Scholar
}

\author{
Rajneesh Talwar, PhD \\ Principal,CGC College Jhanjeri
}

\begin{abstract}
Nowadays in watermarking applications Standard wavelets have certain limitations i.e. poor directional selectivity and property of shift invariance. As a complex number is having together real and imaginary parts, complex wavelet is also having real and imaginary parts which can aid us in overcoming theses limitations .Dual-Tree Complex Wavelet Transform is the latest improvement and in this paper, a watermarking scheme has been projected on the base of Dual Tree Complex Wavelet Transform where DTCWT is initially applied to the host image, and it decomposes that image into eight real and eight imaginary sub bands and then DTCWT is applied to the watermark image, it also decomposes the image into eight real and eight imaginary sub bands. Finally watermarking rule is applied according to fusion principle.
\end{abstract}

\section{Keywords}

Watermarking, Complex Wavelet Transform.

\section{INTRODUCTION}

Digital watermarking [1] is a method that embeds watermark into an image in such a manner that the watermark can be later detected to make an assertion about the originality of the image. Watermarking can be classified as visible or invisible watermarking. Invisible watermark is concealed in the object, in such a manner that it can be sensed by an authorized person only.

Such watermarks are particularly used for author authentication and detecting unauthorized copying. For some applications of the DWT improvements can be made by making use of an extensive wavelet transform instead of a critically-sampled one. There are numerous kinds of extensive DWTs; and the dual-tree complex discrete wavelet transform is one of them. In 2005, Chen [4] suggested a singular value decomposition scheme that is based on components of $\mathrm{D}$ and $\mathrm{U}$ and they proved that the quality of watermarked image is good in their case. In 2007, Patra [5] presented a new digital watermarking scheme, which uses a single key image for extracting different watermarks.

Fusion based watermarking is done by adding the values of the selected sub band with the values of the selected sub band of watermark which is multiplied by an appropriate strength factor. Experimental outcomes demonstrate that the implemented algorithm achieved very high imperceptibility and Robustness against various types of image processing attacks like Gaussian noise, speckle noise, rotation, etc.

\section{LITERATURE SURVEY}

Wavelet transform has more applications in watermarking on images because of its multi resolution property .In [4] S. Tripathi, N. Ramesh proposed that coefficients the DWT of the watermark (logo) are modified using small secondary binary image and the midfrequency coefficients of the cover image. Since the watermark has some features of the host image embedded into it, the security also increases two-fold and the watermark is also protected against any misuse. The technique works for many attacks but for rotation it is tested and valid only for values of limited angle. In [2] W.Lin, J.Sheng proposed a blind digital image watermarking algorithm based on the DWT. The algorithm uses spreadspectrum technique and embeds the watermark into discrete wavelet domain of the original image. The result is improved robustness, imperceptibility, security and reliable to a higher level. In [3] Z.M Lu, \& D.G Xu, a versatile digital image watermarking technique was proposed built on the multistage vector quantizer structure, which can be applied to image authentication and copyright protection. In this case semi fragile watermark and the robust watermark were embedded in VQ stages using diverse techniques, and both of them can also be extracted without the original image. The results show that the algorithm was effective in terms of both robustness as well as fragility.

The algorithms are tested for attacks which include rotation, JPEG Compression but the watermark gets distorted in case of rotation significantly during extraction. In [4] P. Dong, J. G. Brankov proposed watermarking approaches to eliminate the problem of geometric distortions. The first one was a multibit public watermarking scheme which was based on image normalization, and was robust to general geometric attacks. Shortcomings of real valued DWT can be eliminated by the CWT . In recent years, Complex Wavelet Transform has gathered attention in case of image transformation as well as image watermarking.

\subsection{Complex 2-D Dual-Tree Wavelet Transform}

\subsection{Introduction}

The 2-D dual-tree CWT gives rise to wavelets in six different directions, but, in this case there are two wavelets in each direction. In all directions, one of the two wavelets is seen as the real part of a complex-valued 2D wavelet, while the other wavelet is seen as the imaginary part of a complex-valued 2D wavelet. As the complex version is having twice as many wavelets as the real form of the transform, the complex version becomes four times more expansive. The complex 2D dual-tree is realized as four critically-sampled separable 2D DWTs in parallel. But different filters are used along the rows and columns. The CWT can distinguish between two opposing diagonal features which are separate filters oriented at + and $-45^{\circ}$.The redundancy of the CWT has implications on watermark embedding wavelets. The 2-D DT-CWT can be implemented with the help of separable wavelet transforms like 2-D wavelet transform. The 2-D DT-CWT can be implemented with separable wavelet transforms like 2-D wavelet transform. 


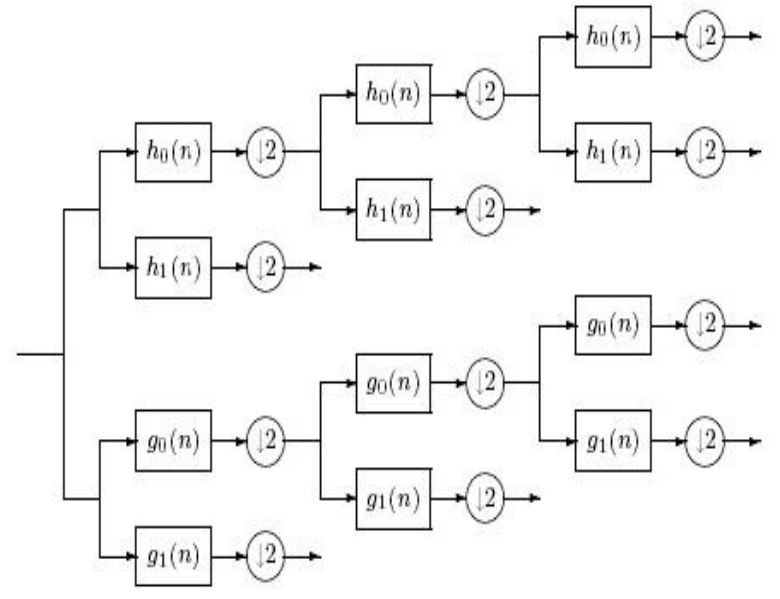

Fig. 1.1 1D Dual Tree Complex Wavelet Transform

1D Dual Tree Complex Wavelet Transform [6] Impulse response of six wavelets of 2-D complex wavelet transform is shown in Fig. 1.2. These six wavelet sub-bands of the 2-D DT-CWT are particularly oriented in in $+15^{\circ},+45^{\circ},+75^{\circ}$,$15^{\circ},-45^{\circ},-75^{\circ}$ direction and captures image information in six directions

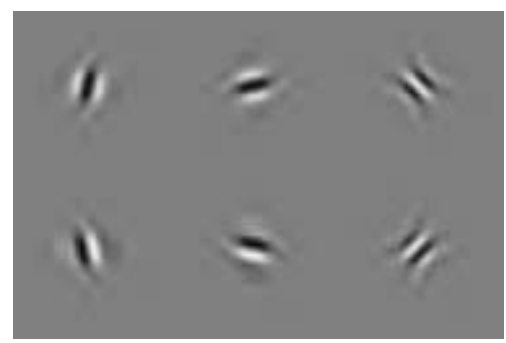

\section{Fig. 1.2 DTCWT coefficients oriented in 6 directions}

DTCWT coefficients oriented in 6 directions DTCWT coefficients oriented in 6 directions The DTCWT uses 2 trees of real filters to create real and imaginary parts of an effectively complex filter the shift invariant property is accomplished with a real DWT by doubling the sampling rate at each level of the tree compared with undecimated wavelet tree, which eliminates down sampling after every level of filtering, DTCWT eliminates down sampling only after first level of filtering..

In standard wavelet there is one scaling function along with one wavelet function. In Complex wavelet we have 2 wavelet functions, and is called a Hilbert pair. In DWT one of the major limitations is that when ever watermarked image suffers rotation, watermark is totally damaged and cannot be retrieved at all but with CWT transform this problem is overcome \& the watermark can be effectively retrieved. The DTCWT coefficients which are obtained from the first filter bank are recognized as the real part and the coefficients that are obtained from the seconds filter are called as the imaginary part. The real part of the image contains less important data compared to the imaginary part which contains more information. Our algorithm puts the watermark into the entire image, that is, the real and imaginary parts together. This is preferable methodology because in case an attack is there while transmitting, it can be easily identified. In other words, if only the real part is used for watermark embedding and an unauthorized person tries to extract the watermark, because the real part contains less information. In the case of using both real and imaginary parts, an attack can be easily identified. The transform is 2-times expansive because for an $\mathrm{N}$-point signal it gives $2 \mathrm{~N}$ DWT coefficients. If the filters in the upper and lower DWTs are the same, then no advantage is gained. However, if the filters are designed is such a way that the sub band signals of the upper DWT can be interpreted as the real part of a complex wavelet transform, and sub band signals of the lower DWT can be interpreted as the imaginary part. In other words, if only the real part is used for embedding the watermark and an unauthorized person tries to take out the watermark, because the real part contains less information. In the case of using both real and imaginary parts, an attack can be identified with ease. If the filters in the upper and lower DWTs are the same, then it makes no difference. But in case the filters are designed is such a way that the sub band signals of the upper DWT are taken as the real part of a CWT [8] and sub band signals of the lower DWT can be taken as the imaginary part. For specially designed sets of filters, the wavelet involved with the upper DWT can be taken as an approximate Hilbert transform of the wavelet linked with the lower DWT. When designed in this manner, the dual-tree complex DWT is almost shift-invariant, in contrast with the critically-sampled DWT. Moreover, the dual-tree complex DWT can be used to realize 2D wavelet transforms where each wavelet is oriented, which is especially useful for image processing. The DTCWT [8] coefficients obtained from the first filter bank are known as the real part and the coefficients obtained from the other filter are known the imaginary part. The real part of the image has less important data. Instead of implanting the watermark into the real part of the image, this algorithm inserts the watermark into the whole image, both the real and imaginary parts. [10].This is desirable because if while transmitting if there is an attack, it can be identified with ease. In other terms it can be said that if only the real part is used for watermark implanting and an unauthorized person attempts to extract the watermark, because the real part contains less information.

\subsection{Block Diagram}

Watermarking Effective image watermarks must be approximately matched to the local spectral characteristics of the image that is being marked, in order to make the mark as invisible as possible (due to perceptual masking) and as robust as possible against denoising attacks. We propose a system which achieves this by watermarking in the complex wavelet domain, since it is very easy, using the DT CWT, to provide the spatially varying spectral characteristics that are required for optimal watermarking. Hence we see that the strengths of the DT CWT stem from its abilities (a) to analyze multidimensional signals unambiguously at multiple scales and directions, and (b) to provide spatially adaptive directional filtering which does not suffer from significant aliasing artifacts, all at modest computational . 


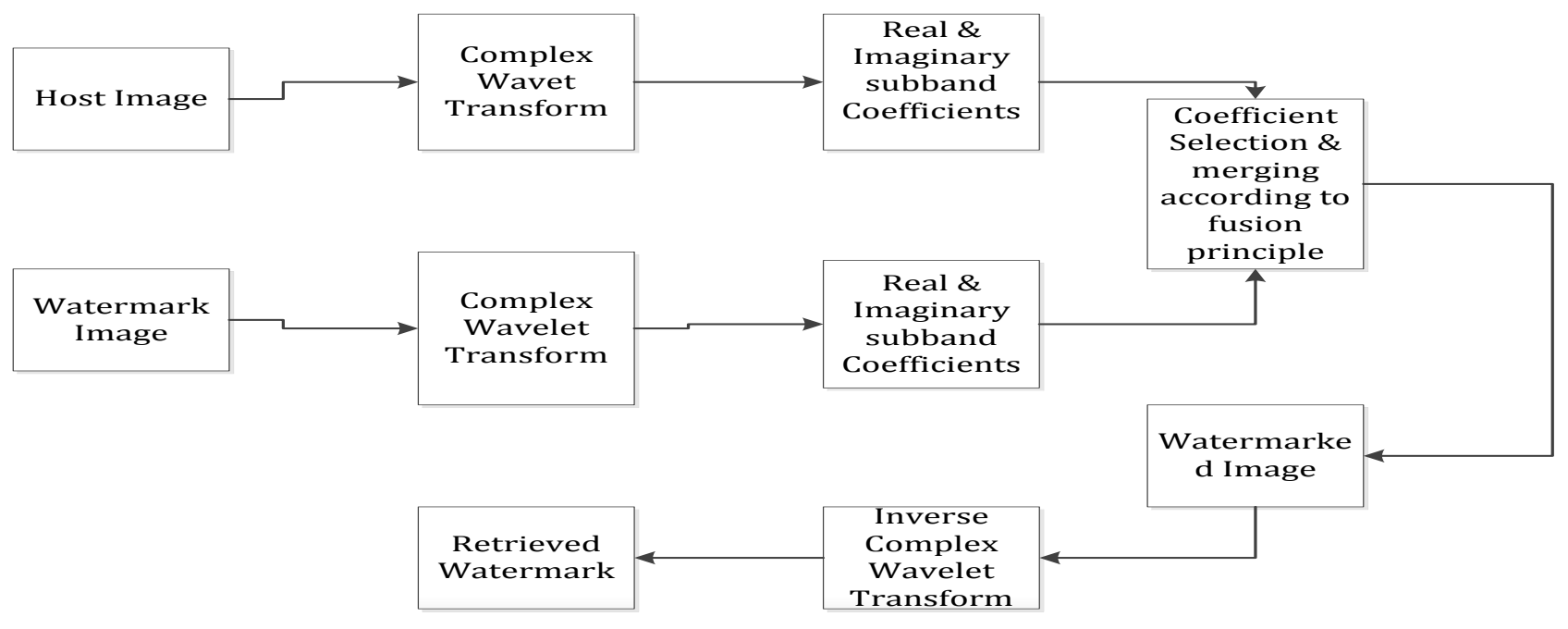

Fig 1.3. Block Diagram of Watermarking Scheme

Dec 1

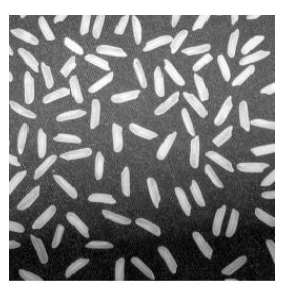

Fig 2 (a) Cover Image

Dec

3

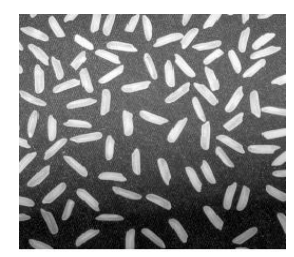

Fig 3(a)Cover Image

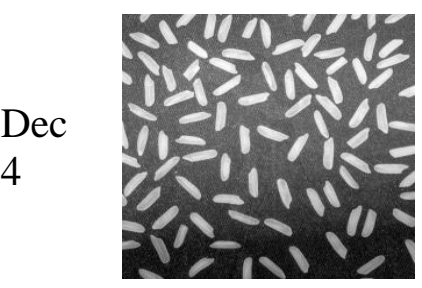

Fig 4(a)Cover Image

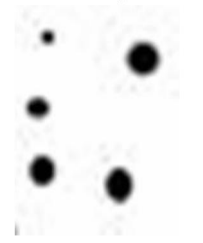

Fig (b) Watermark

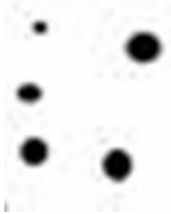

Fig (b) Watermark

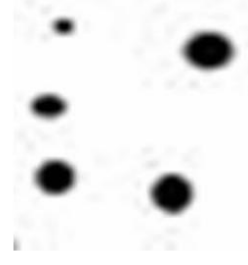

Fig (b) Watermark

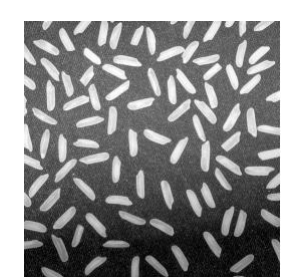

Fig(c)Watermarked Image

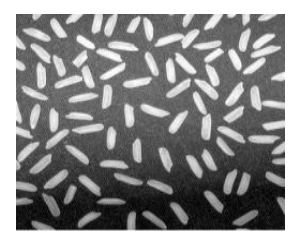

Fig(c)Watermarked Image

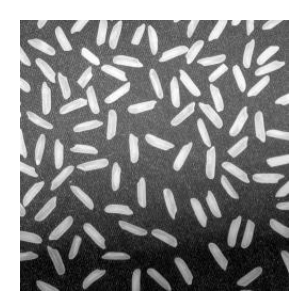

Fig(c)Watermarked Image

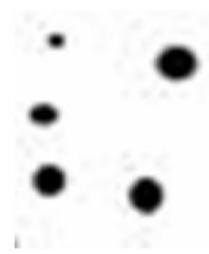

Fig(d)Recovered Watermark

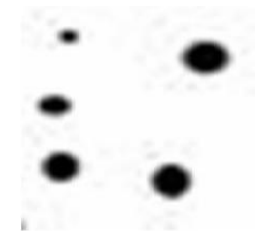

Fig(d)Recovered Watermark

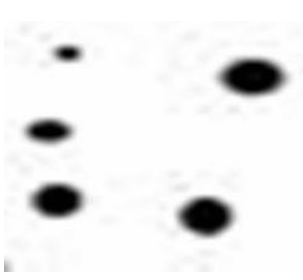

Fig(d)Recovered Watermark 
Dec 5

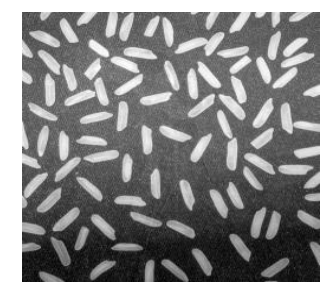

Fig5(a) Cover Image

Dec 6

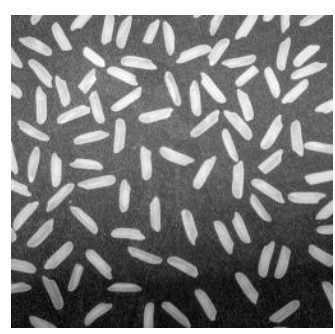

Fig6(a) Cover Image

Dec 7

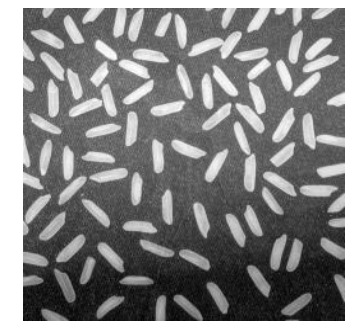

Fig7(a) Cover Image

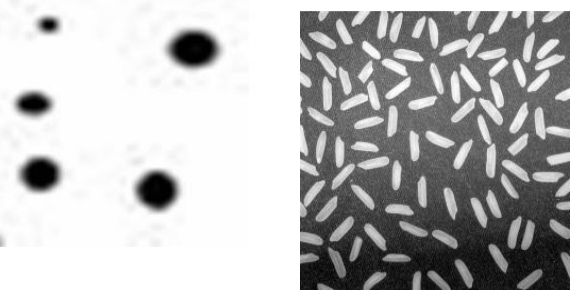

Fig (b) Watermark

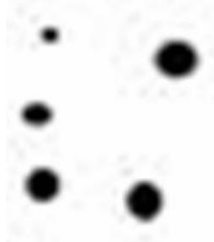

Fig (b) Watermark

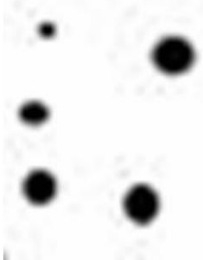

Fig (b) Watermark
Fig(c)Watermarked Image

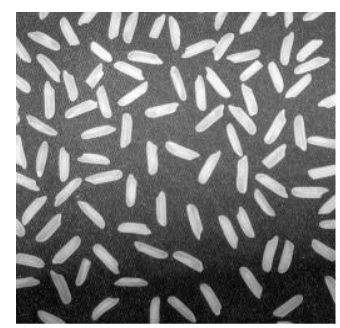

Fig(c)Watermarked Image

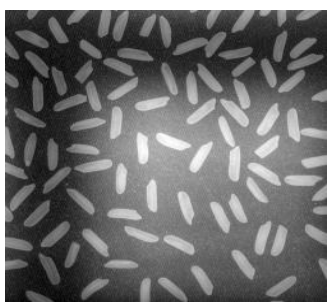

Fig(c)Watermarked Image

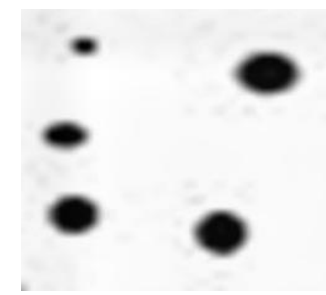

Fig (d) Recovered Watermark

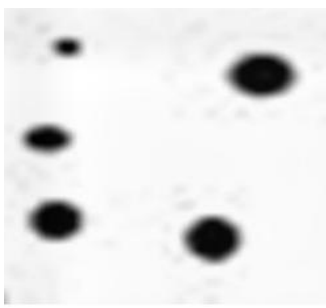

Fig (d) Recovered Watermark

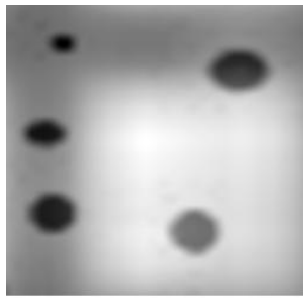

Fig (d) Recovered Watermark

Fig 1.4 Insertion \& retrieval of watermark in different decomposition levels

\begin{tabular}{|c|c|c|c|c|c|}
\hline $\begin{array}{l}\text { Dec } \\
\text { Level }\end{array}$ & $\begin{array}{c}\text { Cover } \\
\text { image }\end{array}$ & Watermark & $\begin{array}{c}\text { PSNR of } \\
\text { watermarked } \\
\text { Image }\end{array}$ & MSE & RMSE \\
\hline 1 & Rice.png & Watermark & $\mathbf{7 5 . 1 4 6 1}$ & $\begin{array}{c}3.9531 e- \\
006\end{array}$ & $\mathbf{0 . 0 0 2 0}$ \\
\hline 2 & Rice.png & Watermark & 73.4595 & $\begin{array}{c}8.5954 e- \\
006\end{array}$ & 0.0029 \\
\hline 3 & Rice.png & Watermark & 69.3207 & $\begin{array}{c}5.7814 e- \\
005\end{array}$ & 0.0076 \\
\hline 4 & Rice.png & Watermark & 63.1292 & 0.0010 & 0.0316 \\
\hline 5 & Rice.png & Watermark & 48.1520 & 0.9903 & 0.9951 \\
\hline 6 & Rice.png & Watermark & 48.1520 & 0.9903 & 0.9951 \\
\hline 7 & Rice.png & Watermark & 32.0675 & $\begin{array}{c}1.6318 e+00 \\
3\end{array}$ & 40.3957 \\
\hline
\end{tabular}

Table 1.1 Table of PSNR,MSE \& RMSE 


\subsection{Experimental Results}

In this experiment, we have inserted watermarks in rice image.Algorithm is tested for no attack, speckle noise,Gaussian noise,Poisson noise\& Rotation Attack. As shown in figure1.4 under no attacks the watermark can be inserted \& retrieved effectively with a PSNR of nearly

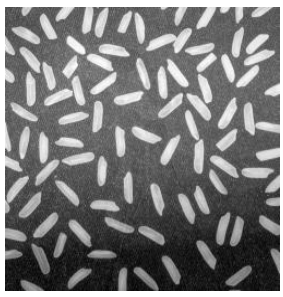

(i)Cover Image

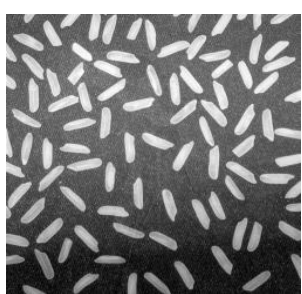

(i)Cover Image

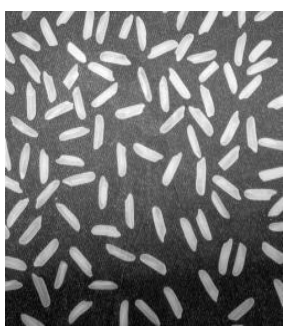

(i)Cover image

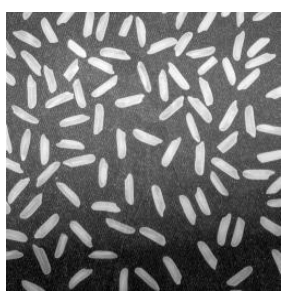

(i)Cover Image

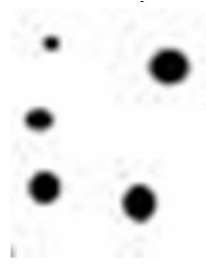

(ii)Watermark

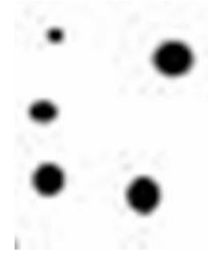

(ii)Watermark

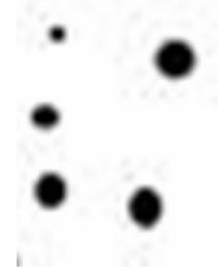

(ii)Watermark

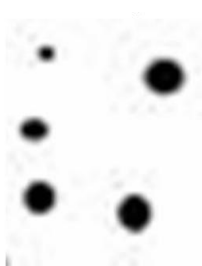

(ii)Watermark

75.After that after applying attacks, we find that the watermark can be retrieved even after the image undergoes attacks with the condition of nearly same imperceptibility. Significantly it works very well for rotation attacks which is a limitation in case of other wavelet transforms.

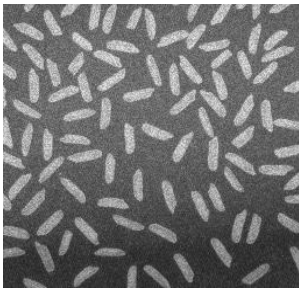

(iii) With Speckle noise

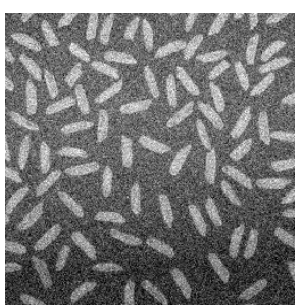

(iii) With Gaussian noise

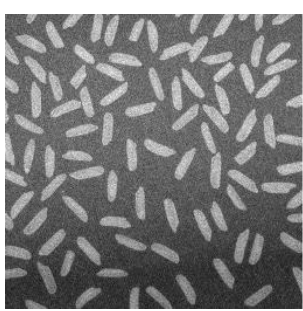

(iii) noise

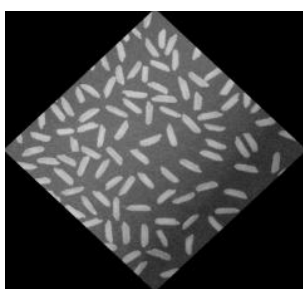

(iii) With Rotation Attack

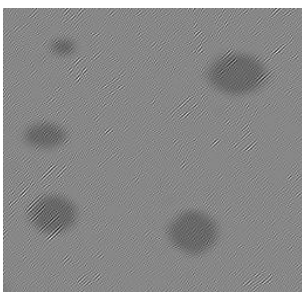

(iv) Retrieved Watermark $P S N R=$

27.352

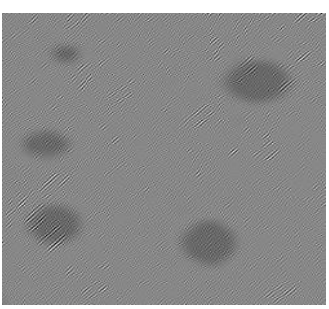

(iv) Retrieved Watermark, $P S N R=$ 27.354

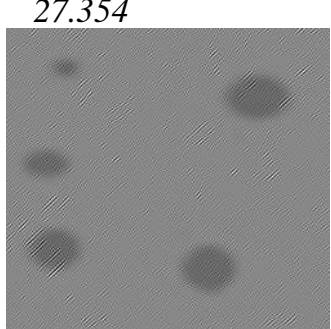

(iv) Retrieved Watermark $P S N R=27.358$

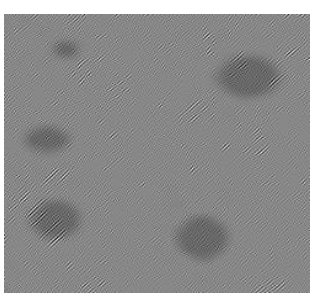

(iv) Retrieved Watermark, $P S N R=$ 27.3502

Fig 1.5 Insertion \& retrieval of watermark with different attacks

\section{CONCLUSION}

A new watermarking algorithm was depicted in this paper, which is centered on Dual Tree Complex Wavelet Transform and fusion principle., as a result, many aspects of property for watermarking were improved. From the experimental result, we conclude that the robustness of proposed algorithm was increased, adding noise, and etc. At the same time, from the comparison we also know that new algorithm is enhanced in imperceptibility, security and reliable separately. 


\section{ACKNOWLEDGMENTS}

We would like to acknowledge the support and guidance offered by IK Gujral Punjab Technical University, Jalandhar, India.

\section{REFERENCES}

[1] "A Perceptually Tuned Watermarking Scheme for Color Images ",Chun-Hsien Chou and Kuo-Cheng Liu IEEE Transactions On Image Processing, Vol. 19, No. 11, November 2010.

[2] "A New DWT \& Multi-Strategy Watermark Embedding Algorithm" W.Lin, J.Sheng 2011 IEEE

[3] "Multipurpose Image watermarking Algorithm Based on Multistage Vector Quantisation” Zhe-Ming Lu, Member, IEEE, Dian-Guo Xu, Member, IEEE, and Sheng-He Sun IEEE Transactions On Image Processing, Vol. 14, No. 6, June 2005 .

[4] "Digital Watermarking Robust to Geometric Distortions", P.Dong, J. G. Brankov, IEEE Transactions On Image Processing, Vol. 14, No. 12, December 2005.

[5] "A DWT based Dual Image Watermarking Technique for Authenticity and Watermark Protection" , Shikha Tripathi1, Nishanth Ramesh2, Bernito A3, Neeraj K J Signal \& Image Processing : An International Journal(SIPIJ) Vol.1, No.2, December 2010.
[6] N. G. Kingsbury, "Shift invariant properties of the DualTree Complex Wavelet Transform", Proc. ICASSP 99, Phoenix, AZ, 16-19, Mar 1999.

[7] "Spread Transform Watermarking for Digital Multimedia Using the Complex Wavelet Domain "A. I. Thompson, A. Bouridane, F. Kurugollu 2007 ECSIS Symposium on Bio-inspired, Learning, and Intelligent Systems for Security

[8] "Rotated Complex Wavelet based Texture Features for Content Based Image Retrieval", Manesh Kokare, P.K. Biswas, and B.N. Chatterji Indian Institute of Technology, Kharagpur Proceedings of the 17th International Conference on Pattern Recognition (ICPR'04) 1051-4651/04 \$20.00 IEEE

[9] N. G. Kingsbury, "Image processing with complex wavelets". Philosophical Transactions Royal Society London A, 1999, 357(9):2543-2560

[10] "Rotated Complex Wavelet Filters", Manesh Kokare, Member, IEEE, P. K. Biswas, Member, IEEE, and B. N. Chatterji IEEE Transactions On Systems, Man, And Cybernetics-Part B: Cybernetics, Vol. 36, No. 6, December 2006 